\title{
PERCEPÇÃO DAS CONSOANTES PLOSIVAS DO PORTUGUÊS BRASILEIRO POR FALANTES NATIVOS DE MANDARIM
}

\section{THE PERCEPTION OF BRAZILIAN PORTUGUESE PLOSIVE CONSONANTS BY MANDARIN NATIVE SPEAKERS}

\section{RESUMO}

Flávia Wen Chun Tso ${ }^{1}$ Cláudia Regina Brescancini ${ }^{2}$

O presente trabalho tem por objetivo investigar a percepção das consoantes plosivas /p, b, t, d, $\mathrm{k}, \mathrm{g} /$ do português brasileiro como língua não nativa (doravante LNN) por falantes nativos de mandarim. As consoantes plosivas do português brasileiro apresentam como traço distintivo a sonoridade; e as do mandarim, a aspiração. Dessa forma, os falantes nativos de mandarim, aprendizes de português, deverão aprender a distinção de sonoridade, já que a distinção por aspiração não tem papel contrastivo nessa língua. Foram aplicados quatro testes de identificação das consoantes em análise, dois com palavras reais e dois com logatomas. Os testes foram aplicados a cinco chineses bilíngues, três do sexo feminino e dois do sexo masculino, com idades compreendidas entre 18 e 30 anos, com níveis de proficiência intermediário e avançado. Os resultados indicaram que os participantes apresentaram melhor desempenho na identificação das consoantes $/ \mathrm{k}, \mathrm{g} /$ e nos testes de identificação que consideraram como estímulos palavras reais.

PALAVRAS-CHAVE: consoantes plosivas; mandarim; percepção da fala.

\section{ABSTRACT}

The present work aims to investigate the perception of Brazilian Portuguese plosive consonants $/ \mathrm{p}, \mathrm{b}, \mathrm{t}, \mathrm{d}, \mathrm{k}, \mathrm{g} / \mathrm{as}$ a non native language (NNL) by native speakers of Mandarin. Plosive consonants in Brazilian Portuguese present voicing as a distinctive feature; and those of mandarin, aspiration. Thus, native speakers of Mandarin, learners of Portuguese, should learn the distinction of voicing, since the distinction of aspiration does not have a contrastive role in Brazilian Portuguese. Four tests of identification of consonants were applied, two with real words and two with non sense words. The tests were applied to five Chinese bilingual participants, three women and two men, aged between 18 and 30, with intermediate and advanced proficiency levels. The results indicated that the participants presented better performance in the identification of the consonants $/ \mathrm{k}, \mathrm{g} /$ and in the identification tests that considered as stimuli real words.

KEY-WORDS: plosive consonants; Mandarin; speech perception

\section{INTRODUÇÃO}

O presente estudo tem por objetivo investigar a percepção das consoantes $/ \mathrm{p} /, / \mathrm{b} /, / \mathrm{t} /$, $/ \mathrm{d} /, / \mathrm{k} /, / \mathrm{g} /$ por falantes nativos de mandarim em processo de aquisição do português brasileiro (doravante PB) como língua não nativa (doravante LNN). Parte-se da constatação de que chineses tendem a apresentar dificuldade na produção e na percepção das consoantes

\footnotetext{
${ }^{1}$ Mestre em Letras pelo Programa de Pós-Graduação em Letras da Escola de Humanidades da PUCRS. email: flavia_tso@hotmail.com

2 Professora Adjunta do Programa de Pós-Graduação em Letras da Escola de Humanidades da PUCRS. email: bresc@pucrs.br
} 
plosivas /p, b, t, d, k, g/ do PB, o que se justifica pelo fato de que o sistema de plosivas do mandarim, composto por $/ \mathrm{p}, \mathrm{p}^{\mathrm{h}}, \mathrm{t}, \mathrm{t}^{\mathrm{h}}, \mathrm{k}, \mathrm{k}^{\mathrm{h}} /$, distingue-se pela aspiração, enquanto que no $\mathrm{PB}$ a distinção se dá pela sonoridade.

De acordo com o Modelo de Aprendizagem da Fala (SLM) e o Modelo de Assimilação Perceptual (PAM e PAM-L2) propostos, respectivamente, por Flege (1995) e por Best e Tyler (2007), a dificuldade quanto à percepção de um contraste varia de acordo com as similaridades e as diferenças entre as propriedades fonéticas dos sons não nativos em relação aos sons da língua nativa. Desse modo, na fase inicial da aprendizagem de uma LNN, os falantes tenderiam a "misturar" os segmentos dessa língua que apresentam características fonéticas e fonológicas semelhantes aos da língua materna (doravante L1). Como as plosivas contrastivas do PB se diferem das plosivas contrastivas do mandarim pelo traço de sonoridade e pela ausência distintiva da aspiração, parte-se da hipótese de que os aprendizes chineses apresentariam dificuldades na hora de distinguir essas consoantes, sobretudo os falantes chineses menos proficientes em português. Por outro lado, os aprendizes com mais exposição à LNN tenderiam a apresentar resultados melhores, já que, de acordo com Flege (1995) e Best (2007), o uso mais ou menos intenso da LNN é relevante para o processo de aquisição.

Este estudo divide-se em três seções, além desta introdução. O sistema de plosivas das duas línguas, PB e mandarim, é apresentado a seguir, assim como também os resultados do estudo de Yang (2014), que motivou algumas das hipóteses específicas que serão testadas. $\mathrm{Na}$ seção 3 é apresentada a metodologia adotada e, na seção 4, são descritos os resultados obtidos. Seguem-se considerações finais e referências.

\section{Mandarim e Português: sistema de plosivas e percepção da fala}

Língua oficial da China e de caráter tonal, o mandarim é a variedade baseada nas normas dialetais da região norte e nordeste do país, principalmente a da capital Pequim. De acordo com Yuan (2001), dentre as sete famílias dialetais ${ }^{3}$ presentes nesse país, o dialeto mandarim e o dialeto Wu são os que possuem mais falantes, ocupando, respectivamente, $70 \%$ e $8 \%$ da população chinesa.

De acordo com Huang e Li (2012), o sistema de plosivas do mandarim, presente em posição inicial de sílaba, é composto por $/ \mathrm{p}, \mathrm{p}^{\mathrm{h}}, \mathrm{t}, \mathrm{t}^{\mathrm{h}}, \mathrm{k}, \mathrm{k} /$, conforme ilustram os pares mínimos apresentados no Quadro 1 a seguir. Da mesma forma que o português, distribui-se por três pontos de articulação, labial, coronal e dorsal, com predominância de consoantes [+anterior] (labiais e coronais). Embora não haja, como no português, a distinção de [voz] nesse sistema, sons plosivos [+voz] se fazem presentes alofonicamente quando os segmentos $/ \mathrm{p} /, / \mathrm{t} / \mathrm{e} / \mathrm{k} / \mathrm{se}$ encontram na posição inicial de uma sílaba constituída por um tom neutro e produzida de forma leve e breve, situação em que a tendência é o falante produzir os sons $[\mathrm{p}]$, $[\mathrm{t}] \mathrm{e}[\mathrm{k}]$ como $[\mathrm{b}]$, $[\mathrm{d}] \mathrm{e}$ [g], como em [wei·ba] 'rabo' (尾巴); [wo`də] 'meu' (我的) e [gə·gə] 'irmão mais velho' (哥哥) . Essas realizações alofônicas apresentadas são explicadas pelo fato de essas sílabas serem produzidas com menos força articulatória e, consequentemente, ocorrer o enfraquecimento dos sons $[\mathrm{p}],[\mathrm{t}] \mathrm{e}[\mathrm{k}]^{2}$. Como o tom neutro ocorre em contextos limitados, realizações dos alofones [b], [d] e [g] são encontradas com baixa frequência na fala cotidiana.

\footnotetext{
3 Dialeto Wu, falado na região sudeste da China, como na província Zhejiang, na cidade Xangai e nas cidades localizadas na região sul da província Jiangsu; o dialeto Xiang, falado principalmente na província Hunan; o dialeto Gan, falado principalmente na província Jiangxi; o dialeto Hacá, falado em Fujian, Taiwan e Hong Kong; o dialeto Yue, falado nas províncias Guangdong e Hainan e em Hong Kong e Macau e o dialeto Min, cujos falantes concentram-se principalmente nas províncias como Fujian, Hainan, Taiwan e nas cidades localizadas na região do sul da província Zhejiang.

2 O papel da força consonantal é explicado por meio da estipulação de uma escala universal por Jespersen (1904 apud CLEMENTS, 1990), de acordo com a qual oclusivas surdas $>$ continuas surdas e oclusivas sonoras $>$ contínuas sonoras $>$ nasais $>$ líquidas $>$ glides.
} 
Quadro 1 - Pares Mínimos envolvendo Plosivas em Mandarim

\begin{tabular}{|c|c|c|}
\hline Pares mínimos & Logogramas & Significados \\
\hline$/ \mathrm{pa}^{35} / 4 / \mathrm{p}^{\mathrm{h}} \mathrm{a}^{35} / / \mathrm{ma}^{35} / / \mathrm{fa}^{35} /$ & 拔爬 麻 罚 & arrancar, subir, linho, punir \\
\hline$/ \mathrm{tu}^{51} / / \mathrm{t}^{\mathrm{h}} \mathrm{u}^{51} / / \mathrm{nu}^{51} / / \mathrm{lu}^{51} /$ & 肚 兔 怒 路 & barriga, coelho, irritar-se, rua \\
\hline$/ \mathrm{k}^{55} / / \mathrm{k}^{\mathrm{h}} \mathrm{\partial}^{55} / / \mathrm{x} \partial^{55} /$ & 歌 科 喝 & música, disciplina, beber \\
\hline
\end{tabular}

A percepção das consoantes plosivas do Português, a partir da produção da variedade europeia, por falantes do mandarim foi examinada por Yang (2014), com base no Modelo de Aprendizagem da Fala (SLM) (FLEGE, 1995) e no Modelo de Assimilação Perceptiva (PAM-L2) (BEST; TYLER, 2007).

A amostra analisada contou com 54 participantes, divididos em três grupos: dois grupos experimentais formados por falantes nativos de mandarim e um grupo de controle. Os dois primeiros grupos foram subdivididos em Grupo 1 (G1) e Grupo 2 (G2), sendo que o G1 incluiu 16 participantes (três homens e 13 mulheres, com idade média de 21,94 anos); e o G2 incluiu 20 participantes (seis homens e 14 mulheres, com idade média de 19,70 anos). Todos eram estudantes de língua portuguesa, variedade europeia (doravante PE), na China e participaram de um programa de intercâmbio em Portugal; no entanto, o G1 compreendeu os alunos que já cursaram três anos de curso de PE e que moraram em torno de 10 a 12 meses em Portugal; e o G2 compreendeu os alunos do primeiro ano do curso de PE e que recém tinham chegado a Portugal. O grupo de controle (G3) incluiu 18 estudantes universitários, nativos de PE e residentes em Portugal, com idade média de 18,83 anos.

Foram aplicados dois testes de percepção ao G1 e ao G2: um teste de identificação, no qual o participante teve de identificar o estímulo apresentado a partir de duas alternativas de respostas ou de um conjunto de alternativas; e um teste de discriminação, no qual o participante teve de indicar se o par de estímulos apresentados eram iguais ou diferentes. Para isso, foram gravados 48 estímulos por três falantes do G3. Os estímulos mantiveram as consoantes /b, d, g, $\mathrm{p}, \mathrm{t}, \mathrm{k} /$ no mesmo contexto vocálico, seguidas pelas vogais /a/ e /o/ na posição tônica e átona e sempre na posição de ataque silábico inicial ou medial. Todos os estímulos apresentaram a estrutura CVCV ou CVCV(C).

Os resultados obtidos revelaram diferenças significativas na percepção dos segmentos /b, $\mathrm{d}, \mathrm{g}, \mathrm{p}, \mathrm{t}, \mathrm{k} / \mathrm{entre}$ os falantes nativos de PE e os falantes nativos de mandarim do G1 e G2; porém, não entre os dois grupos de falantes chineses, já que ambos mostram dificuldades na percepção das consoantes plosivas em $\mathrm{PB}$.

Além disso, comprovou-se que a imersão linguística exerce uma influência parcialmente positiva, mas não tão significativa. Os participantes do G1, que tiveram mais contato com o PE em um ambiente natural, apresentaram facilidade maior na identificação dos segmentos $/ \mathrm{t} / \mathrm{e}$ /k/; porém, de modo geral, não houve diferença relevante entre esse grupo e o G2. O estudo mostrou ainda que os participantes apresentaram maior dificuldade em perceber as consoantes bilabiais /b/, /d/ e /p/ e menos as consoantes /g/, /k/ e /t/ e que os dois grupos de falantes nativos de mandarim não alcançaram uma média alta de acerto no teste de discriminação dos pares $/ \mathrm{b}-\mathrm{p} /, \mathrm{d}-\mathrm{t} / \mathrm{e} / \mathrm{g}-\mathrm{k} /$, sendo o par velar /g- $\mathrm{k} /$, dentre os pares contrastivos, o mais difícil de ser percebido, seguido por $/ \mathrm{b}-\mathrm{p} /$. Quanto à sonoridade, o estudo apontou que os participantes conseguiram discriminar melhor as consoantes [+voz] do que as [-voz], uma vez

\footnotetext{
${ }^{4}$ Em mandarim, uma língua tonal, existem quatro tons lexicais, indicados pelos valores tonais 55 (tom constante), 35 (tom crescente), 214 (tom decrescente e crescente) e 51 (tom decrescente) (DUANMU, 2007).
} 
que não há consoantes plosivas sonoras em mandarim, o que parece tornar esse grupo mais saliente à percepção.

No teste de identificação, o G1 mostrou um desempenho significativamente melhor do que o G2 na identificação das plosivas surdas, o que parece comprovar a influência positiva da imersão linguística; no entanto, levando em consideração o seu desempenho no teste de discriminação dos pares contrastivos, essa afirmação não pode ser sustentada.

Com base no estudo de Yang (2014) e nos resultados obtidos para o PE, a pesquisa em tela, que investiga a percepção das consoantes plosivas em PB por falantes nativos de mandarim, pretende: (i) identificar os possíveis elementos condicionadores, de natureza linguística e/ou social, da percepção esperada; (ii) avaliar o papel do conhecimento prévio do significado das palavras; (iii) investigar a influência da vogal núcleo/a/ e /o/ posterior às plosivas /p, b, d, t, k, $\mathrm{g} /$.

Na próxima seção será apresentada a metodologia do estudo proposto.

\section{Método}

Participaram deste estudo cinco participantes chineses com idade entre 20 e 30 anos, sendo três do sexo feminino e dois do sexo masculino. Dentre eles, dois são alunos da Universidade de Comunicação da China (CUC), recém-chegados a Porto Alegre-RS e participantes do Programa de Português para Estrangeiros (doravante PPE) da Universidade Federal do Rio Grande do Sul (UFRGS); três são chineses que já residiram em Porto Alegre por mais de um ano e que já foram alunos do PPE da UFRGS. Todos os participantes selecionados nesta pesquisa são falantes nativos da região norte da China. O Quadro 2 a seguir apresenta as informações referentes a cada um dos participantes desta pesquisa.

\begin{tabular}{|c|c|c|c|c|c|c|}
\hline \multicolumn{7}{|c|}{ Quadro 2 - Classificação dos participantes } \\
\hline Participante & Cidade Natal & Sexo & Idade & $\begin{array}{c}\text { Tempo no } \\
\text { Brasil } \\
\text { (meses) }\end{array}$ & $\begin{array}{c}\text { Tempo de contato } \\
\text { com PB } \\
\text { (meses) }\end{array}$ & Nível - PB \\
\hline W & $\begin{array}{l}\text { Jinzhou, } \\
\text { Liaoning }\end{array}$ & Feminino & 29 & 96 & 96 & Avançado \\
\hline $\mathrm{C}$ & $\begin{array}{l}\text { Dandong, } \\
\text { Liaoning }\end{array}$ & Feminino & 20 & 2 & 34 & $\begin{array}{c}\text { Intermediário para } \\
\text { avançado }\end{array}$ \\
\hline $\mathrm{H}$ & Pequim & Feminino & 20 & 2 & 34 & Intermediário \\
\hline $\mathrm{F}$ & Hejin, Shanxi & Masculino & 23 & 36 & 44 & $\begin{array}{c}\text { Intermediário para } \\
\text { avançado }\end{array}$ \\
\hline $\mathrm{V}$ & Tianjin & Masculino & 26 & 30 & 30 & Avançado \\
\hline
\end{tabular}

O nível de proficiência de cada participante foi registrado de acordo com o indicado pelo curso do PPE da UFRGS. Conforme a descrição encontrada no site oficial ${ }^{5}$, os cursos do PPE são divididos em cinco níveis: básico I, básico II, intermediário I, intermediário II e avançado.

Com relação aos aspectos geográficos das cidades listadas no Quadro 2, Jinzhou, Dandong, Pequim, Hejin e Tianjin são cidades vizinhas, localizadas na região norte da China, conforme apresentado na Figura 1 a seguir. Ressalta-se que Jinzhou e Dandong pertencem à província Liaoning, indicada pela cor verde no mapa, e Hejin, à província Shanxi, indicada pela cor azul no mapa.

\footnotetext{
${ }^{5}$ Disponível em: http://www.ufrgs.br/ppe/cursos
} 
Figura 1 - Regiões da China consideradas neste estudo

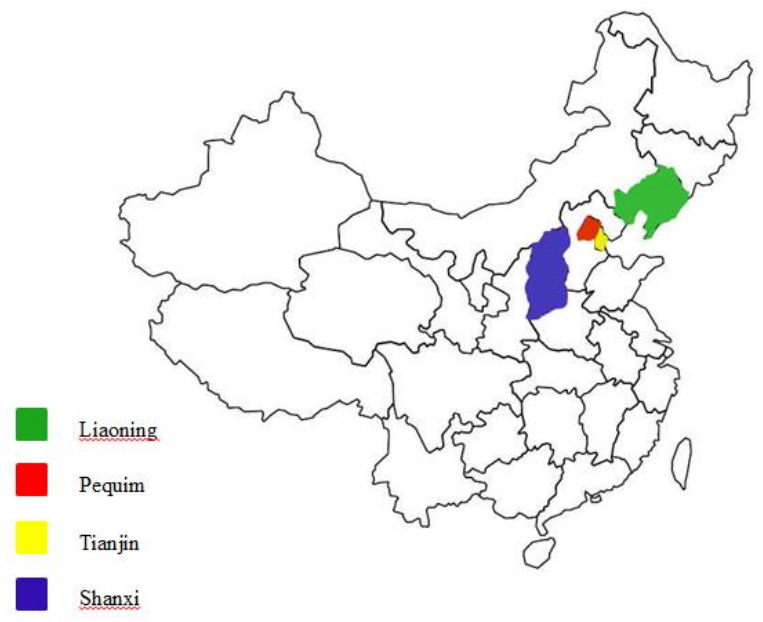

Fonte: Tso (2016)

A fim de responder as questões de investigação deste trabalho, apresentadas na seção 2 anterior, foram propostos quatro testes de identificação. Com o objetivo de identificar o papel do conhecimento do significado de palavra e o da coarticulação entre as plosivas /p, b, t, d, k e $\mathrm{g} / \mathrm{e}$ as vogais /a/ e /o/ no processo de percepção das consoantes plosivas em PB por falantes nativos de mandarim, a construção dos estímulos foi feita conforme os seguintes critérios:

a) Os estímulos abrangem não somente as palavras reais, mas também logatomas, a fim de que seja possível testar a relevância do conhecimento prévio das palavras no processo de identificação das plosivas por falantes chineses;

b) As palavras reais selecionadas são as que aparecem com mais frequência na fala cotidiana para garantir, o máximo possível, a familiaridade dos participantes com os vocábulos;

c) As plosivas /p, b, t, d, k, g/ são inseridas sempre no mesmo contexto fonológico: adjacentes à

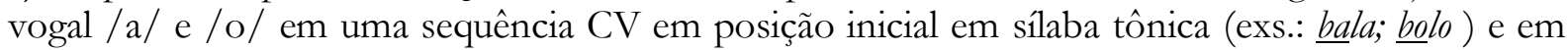
sílaba átona (exs.: batata; bochecha) e em posição medial em sílaba átona (exs.: boba; rabo). Os estímulos possuem no máximo três sílabas ${ }^{6}$.

Os estímulos construídos são apresentados nos Quadros 3 e 4 a seguir.

Quadro 3 - Palavras Reais como Estímulos

\begin{tabular}{|c|c|c|c|c|c|}
\hline \multicolumn{2}{|c|}{ PLOSIVAS NA POSIÇÃO INICIAL } & \multicolumn{2}{c|}{$\begin{array}{l}\text { PLOSIVAS NA POSIÇÃO MEDIAL } \\
\text { INTERVOCÁLICA }\end{array}$} \\
\hline SÍLABA TÓNICA & SÍLABA ÁTONA & \multicolumn{2}{c|}{ SÍLABA ÁTONA } \\
\hline bala & bolo & batata & bochecha & boba & rabo \\
\hline pata & povo & palhaço & possuir & sopa & sapo \\
\hline data & doce & datar & dobrar & roda & lado \\
\hline taxa & topo & tabaco & tocar & gata & gato \\
\hline galo & gota & galinha & goleiro & ruga & lago \\
\hline carro & coco & cabeça & colar & faca & saco \\
\hline
\end{tabular}

\footnotetext{
${ }^{6}$ Os participantes deste estudo, durante a aplicação dos instrumentos de percepção, não apresentaram dificuldade na identificação das sílabas.
} 
Quadro 4 - Logatomas como Estímulos

\begin{tabular}{|c|c|c|c|c|c|}
\hline \multicolumn{4}{|c|}{ PLOSIVAS EM POSIÇÃO INICIAL } & \multirow{2}{*}{\multicolumn{2}{|c|}{$\begin{array}{c}\text { PLOSIVAS EM } \\
\text { POSIÇÃO MEDIAL } \\
\text { INTERVOCÁLICA } \\
\text { SÍLABA ÁTONA }\end{array}$}} \\
\hline \multicolumn{2}{|c|}{ SÍLABA TÔNICA } & \multicolumn{2}{|c|}{ SÍLABA ÁTONA } & & \\
\hline bapa & bôpo & badata & bodata & paba & pabo \\
\hline paba & pôpo & padata & podata & bapa & gapo \\
\hline dapa & dôdo & dadata & dodata & gada & pado \\
\hline tada & tôbo & tadata & todata & fata & sato \\
\hline gapa & gôpo & gadata & godata & gaga & tago \\
\hline cafa & côgo & cadata & codata & gaca & faco \\
\hline
\end{tabular}

Os estímulos apresentados acima foram distribuídos em quatro testes de identificação. Foram gravados por um falante nativo de PB do sexo masculino, em uma sala sem ruídos no entorno. Os instrumentos utilizados para a gravação foram um microfone cardioide (SHURE SM58) e um módulo externo de captura de som (EDIROL UA-25).

A elaboração dos testes foi desenvolvida com o uso do Software Praat 6.0.21 (BORESMA; WEENINK, 2016). Foi solicitado a cada participante que identificasse a consoante plosiva do estímulo apresentado de acordo com o enunciado do teste. Os quatros experimentos são caracterizados do seguinte modo:

a) Teste de Identificação 1: é apresentado auditivamente ao participante estímulos compostos de palavras reais; o participante tem de identificar, de acordo com o enunciado do teste (como apresentado na Figura 2), a alternativa correta dentre um par de possibilidades de resposta. As opções de respostas são constituídas somente por palavras reais, conforme apresentado na Figura 3.

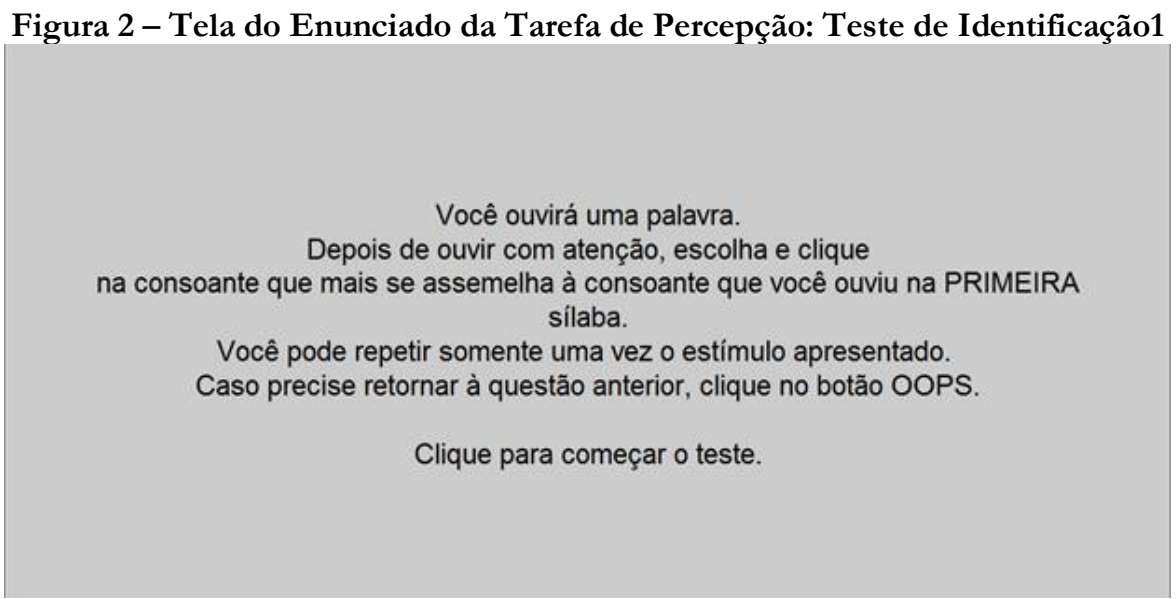

Fonte: Tso (2016), por meio do software Praat (BORESMA; WEENINK, 2016)

\footnotetext{
${ }^{7}$ Disponível em: http://www.fon.hum.uva.nl/praat/download_win.html
} 
Figura 3 - Tela da Tarefa de Percepção: Teste de Identificação1 Escolha a opção que apresenta, na sua PRIMEIRA sílaba, a consoante que mais se assemelha à consoante que você ouviu na PRIMEIRA silaba do estimulo.

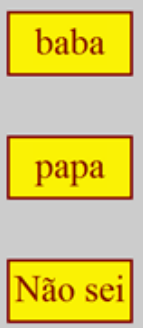

Repetir

Fonte: Tso (2016), por meio do software Praat (BORESMA; WEENINK, 2016)

b) Teste de Identificação é apresentado ao participante auditivamente um estímulo composto de logatoma; as opções de respostas são formadas somente por logatomas, conforme apresentado na Figura 4 e na Figura 5 a seguir.

Figura 4 - Tela do Enunciado da Tarefa de Percepção: Teste de Identificação 2

Você ouvirá uma pseudopalavra.

Depois de ouvir com atenção, escolha e clique

na palavra que apresenta, na sua SEGUNDA silaba, a consoante que mais se assemelha

à consoante que você ouviu na SEGUNDA silaba do estímulo.

Você pode repetir somente uma vez o estímulo apresentado.

Caso precise retornar à questão anterior, clique no botão OOPS.

Clique para começar o teste.

Fonte: Tso (2016), por meio do software Praat (BORESMA; WEENINK, 2016)

Figura 5 - Tela da Tarefa de Percepção: Teste de Identificação 2

Escolha a opção que apresenta, na sua SEGUNDA silaba, a consoante que mais se

assemelha à consoante que você ouviu na SEGUNDA silaba do estímulo

zada

zata

Não sei 
c) Teste de Identificação 3: trata-se de teste de identificação com as palavras reais, porém, as opções apresentadas como respostas são constituídas somente por segmentos consonantais isolados, conforme apresentado na Figura 6 e 7 abaixo.

Figura 6 - Tela da Tarefa de Percepção: Teste de Identificação 3

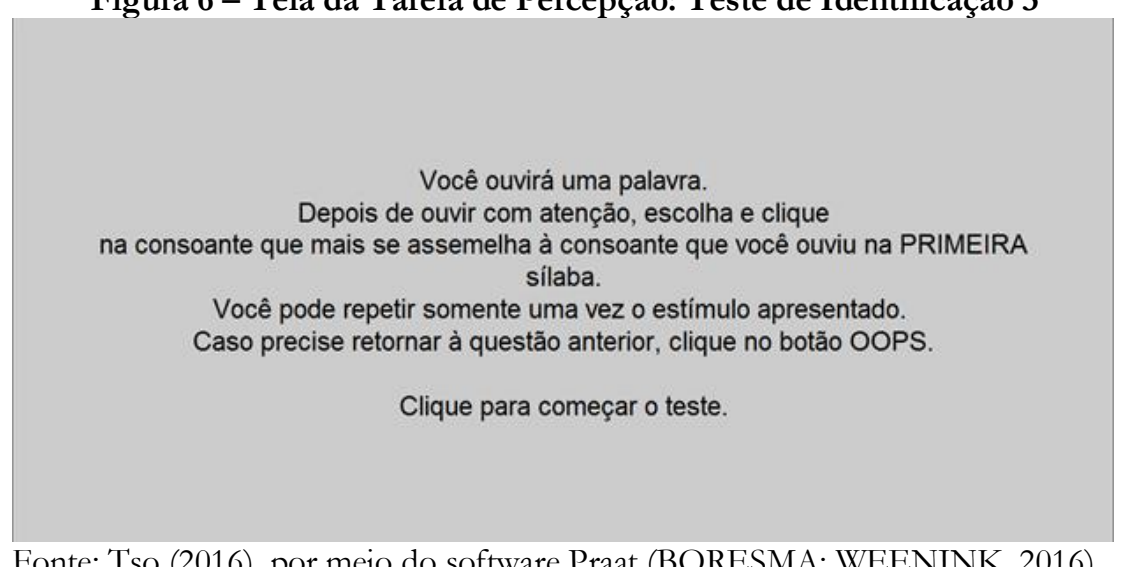

Fonte: Tso (2016), por meio do software Praat (BORESMA; WEENINK, 2016)

Figura 7 - Tela da Tarefa de Percepção: Teste de Identificação 3

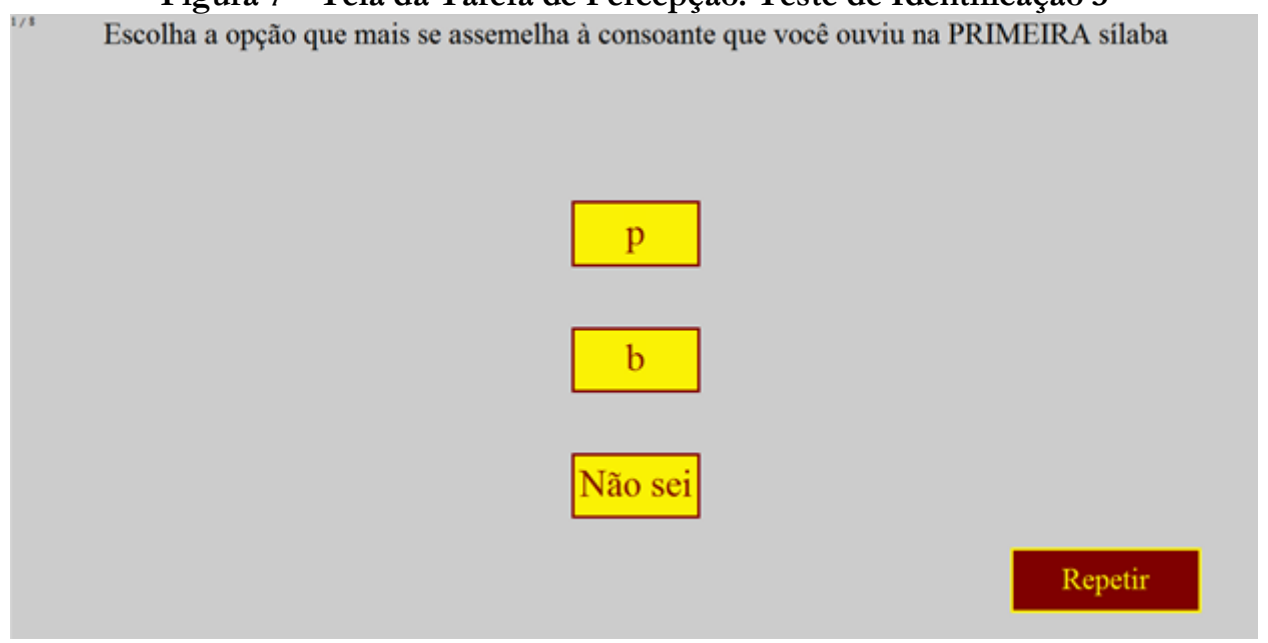

Fonte: Tso (2016), por meio do software Praat (BORESMA; WEENINK, 2016)

d) Teste de Identificação 4: trata-se de teste de identificação com os logatomas; no entanto, diferentemente do Teste 2 , as opções apresentadas como respostas são constituídas somente por segmentos consonantais isolados. As Figuras 6 e 7 anteriores servem também para exemplificar a tela do Praat neste teste.

Ressalta-se que a elaboração do Teste de Identificação 2 e do Teste de Identificação 4 é justificada pela necessidade de examinar se a ausência/presença de outros segmentos linguísticos apoiaria ou não o processo perceptual das plosivas em PB por falantes chineses.

A coleta foi realizada no mês de outubro de 2016. Os participantes foram atendidos individualmente em ambiente silencioso e favorável à aplicação e à realização dos testes de percepção. A forma de obtenção dos dados foi desenvolvida em quatro etapas, sendo que a primeira constou da leitura e assinatura do Termo de Consentimento Livre e Esclarecido em português e em mandarim; a segunda, da aplicação de um questionário sobre o uso da L1 e da LNN, elaborado com base em Silva (2014); a terceira, da aplicação dos experimentos de percepção, com o uso do notebook (ASUS A43S) e fone de ouvido (SONY MDR-NC8); na última etapa foi aplicado um questionário sobre o grau de dificuldade enfrentado pelo participante durante a realização dos testes. O participante teve de destacar também as palavras desconhecidas e escrever ao lado dessas as associações feitas com outras palavras em PB. 
Salienta-se que, na terceira etapa, para evitar o efeito da ordem nos testes de percepção, a forma da aplicação dos testes e da apresentação dos estímulos foi aleatória, por meio do uso de script apropriado.

Durante a aplicação dos testes, foi permitido ao participante repetir o estímulo por somente uma vez e retornar ao estímulo anterior para refazer a questão caso tenha se enganado. Para todos os testes, as opções de respostas incluíam também a alternativa Não Sei, conforme apresentado nas Figuras 5, 6 e 7 anteriores. O participante pode também fazer uma pequena pausa entre cada um desses testes.

Ao final de cada um dos testes, o script gerou uma tabela de resultados com a identificação do nome do script, do nome do estímulo apresentado, da resposta escolhida pelo participante e do tempo de reação, como apresentado na Figura 8 a seguir.

Figura 8 - Resultado fornecido pelo Script

\begin{tabular}{|c|c|c|c|}
\hline $\begin{array}{l}\text { ubject } \\
\text { ala } \\
\text { atata } \\
\text { ochecha }\end{array}$ & $\begin{array}{l}\text { stimulus } \\
\text { b_bala_1 } \\
\text { b_batata_1 } \\
\text { a b_bolo_1 } \\
\text { p_palhaço_1 } \\
\text { p_pata_1 } \\
\text { p_possuir_1 } \\
\text { p_povo_1 }\end{array}$ & $\begin{array}{l}\text { response } \\
\text { baba } \\
\text { pacote } \\
\text { cha_1 } \\
\text { bobo } \\
\text { pacote } \\
\text { papa } \\
\text { poluir } \\
\text { poco }\end{array}$ & \begin{tabular}{l}
\multicolumn{1}{c}{ reactiontime } \\
2.899713258640986 \\
0.19535180924867745 \\
pobreza 0.13260203726531472 \\
0.16492804246445303 \\
0.011791379376518307 \\
-0.06915660736922291 \\
0.20038919360740692 \\
0.04068371705579921
\end{tabular} \\
\hline
\end{tabular}

Fonte: Tso (2016), por meio do software Praat (BORESMA; WEENINK, 2016)

As variáveis independentes de natureza linguística e social, que podem ou não influenciar o desempenho dos participantes chineses nos testes de identificação aplicados nesta pesquisa, são apresentadas a seguir, assim como também os fatores que as compõem:

a) Tipo de Teste de Identificação das Plosivas: teste palavras reais e palavras reais; teste palavras reais e segmentos; teste logatomas e logatomas; teste logatomas e segmentos.

b) Tonicidade: sílaba tônica em posição inicial; sílaba átona em posição inicial; sílaba átona em posição medial.

c) Vogal Seguinte: vogal /a/; vogal /o/.

d) Ponto de Articulação da Consoante Plosiva: alveolares (/t/ e /d/); bilabiais $(/ \mathrm{p} / \mathrm{e}$ /b/); velares $(/ \mathrm{k} / \mathrm{e} / \mathrm{g} /)$.

e) Participantes: W; C; F; V; H.

f) Nível de Proficiência em PB: nível intermediário; nível intermediário para avançado; nível avançado.

g) Tempo de Contato com PB: 30 meses; 34 meses; 44 meses; 96 meses.

h) Tempo de Residência no Brasil: dois meses; 30 meses; 36 meses; 96 meses.

A fim de se verificar as variáveis independentes que condicionam a percepção das plosivas do PB por falantes chineses, foi utilizado o cálculo de regressão logística performado pelo programa Rbrul (JOHNSON, 2016). Foi possível obter o percentual de acerto para cada um dos testes e as variáveis que se mostraram significativas para a identificação das plosivas do PB pelos chineses, caso em que os resultados são oferecidos em termos de peso relativo, um valor que varia de 0,0 e 1,0, e de log-odds, que pode ser um valor positivo ou negativo. $O$ valor de peso relativo maior do que 0,50 é indicativo de favorecimento à identificação das plosivas; caso o peso relativo seja inferior a 0,50, ocorre o contrário. Valores positivos de log-odds indicam favorecimento e negativos, desfavorecimento.

$\mathrm{Na}$ seção seguinte são descritos os resultados obtidos nos quatro testes aplicados. 


\section{Resultados}

A computação estatística conduzida pelo programa Rbrul, a partir do cálculo de regressão logística, apontou que a percepção das plosivas no PB por falantes de mandarim é condicionada pelas variáveis Tipo de Teste de Identificação das Plosivas e Ponto de Articulação da Consoante Plosiva.

Os resultados apresentados na Tabela 1 a seguir indicam que os participantes chineses tiveram facilidade maior na identificação dos segmentos oclusivos nos dois testes em que os estímulos foram compostos por palavras reais, com peso relativo de 0,696 e de 0,611, respectivamente. Isso mostra que o tipo de teste que considera palavras familiares aos participantes, palavras reais, favorece a identificação das consoantes plosivas. Dentre os dois testes, os participantes apresentaram maior probabilidade de acerto no teste que apresentou uma palavra real como estímulo e palavras reais como opções de respostas. Os testes que revelaram probabilidades mais baixas de acerto foram os testes com logatomas, com pesos relativos abaixo de 0,5 , principalmente aquele que apresentou somente os segmentos isolados como alternativas de respostas, com peso de 0,275 (log-odds de -0,971).

Tabela 1 - Tipos de Testes de Identificação das Plosivas: respostas esperadas

\begin{tabular}{c|c|c|c|c}
\hline Fator & $\begin{array}{c}\text { Total de } \\
\text { Ocorrências }\end{array}$ & $\begin{array}{c}\text { Porcentagem de } \\
\text { Aplicação }\end{array}$ & $\begin{array}{c}\text { Log- } \\
\text { odds }\end{array}$ & $\begin{array}{c}\text { Peso } \\
\text { Relativo }\end{array}$ \\
\hline $\begin{array}{c}\text { Palavras Reais } \\
\text { e Palavras Reais }\end{array}$ & 180 & $89,4 \%$ & 0,827 & 0,696 \\
\hline $\begin{array}{c}\text { Palavras Reais } \\
\text { e Segmentos }\end{array}$ & 180 & $85,6 \%$ & 0,453 & 0,611 \\
\hline $\begin{array}{c}\text { Logatomas } \\
\text { e Logatomas }\end{array}$ & 179 & $74,3 \%$ & $-0,309$ & 0,423 \\
\hline $\begin{array}{c}\text { Logatomas e } \\
\text { Segmentos }\end{array}$ & 180 & $61,1 \%$ & $-0,971$ & 0,275 \\
\hline \hline
\end{tabular}

Ainda na Tabela 1, observa-se que tanto nos testes com palavras reais, quanto nos de logatomas, os que apresentavam somente segmentos isolados como alternativas de respostas foram os testes comparativamente com menores probabilidades de acerto, isto é, os que se mostraram mais difíceis para os participantes, o que coincidiu com as respostas dos próprios participantes com relação ao segundo questionário aplicado. Todos tiveram mais dificuldade no Teste de Identificação 2 e no Teste de Identificação 4.

$\mathrm{Na}$ Tabela 2, a seguir, foi examinado o papel da variável de Ponto de Articulação da Consoante na percepção das plosivas em análise. Percebe-se que as consoantes velares $(/ \mathrm{k} / \mathrm{e}$ $/ \mathrm{g} /$ ) exercem papel favorecedor para a identificação dos segmentos oclusivos, com peso relativo 0,597. As consoantes bilabiais $(/ \mathrm{p} / \mathrm{e} / \mathrm{b} /)$ apresentam peso relativo ao redor do ponto neutro, de 0,474, mas log-odds negativo (de -0,106), indicativo, portanto, de papel desfavorecedor, assim como também o obtido para as alveolares $(/ \mathrm{t} / \mathrm{e} / \mathrm{d} /)$, com peso relativo de 0,428 e log-odds de 0,289 . 
Tabela 2 - Ponto de Articulação da Consoante

\begin{tabular}{c|c|c|c|c}
\hline Fator & $\begin{array}{c}\text { Total de } \\
\text { Ocorrências }\end{array}$ & $\begin{array}{c}\text { Porcentagem de } \\
\text { Aplicação }\end{array}$ & $\begin{array}{c}\text { Log- } \\
\text { odds }\end{array}$ & $\begin{array}{c}\text { Peso } \\
\text { Relativo }\end{array}$ \\
\hline $\begin{array}{c}\text { Velar } \\
(/ \mathrm{k} / \mathrm{e} / \mathrm{g} /)\end{array}$ & 240 & $83,3 \%$ & 0,395 & 0,597 \\
\hline $\begin{array}{c}\text { Bilabial } \\
(/ \mathrm{p} / \mathrm{e} / \mathrm{b} /)\end{array}$ & 240 & $76,2 \%$ & $-0,106$ & 0,474 \\
\hline $\begin{array}{c}\text { Alveolar } \\
(/ \mathrm{t} / \mathrm{e} / \mathrm{d} /)\end{array}$ & 239 & $73,2 \%$ & $-0,289$ & 0,428 \\
\hline \hline TOTAL & 719 & $77,6 \%$ & \multicolumn{2}{|}{} \\
\hline \hline
\end{tabular}

Fonte: Tso (2016), por meio do programa computacional Rbrul (JOHNSON, 2016)

O resultado anterior, expresso na Tabela 2, coincide com o trabalho de Yang (2014), relatado na seção 2 deste estudo, com relação ao melhor desempenho obtido pelos participantes na identificação de $/ \mathrm{k}, \mathrm{g} / \mathrm{do} \mathrm{PE}$ por dois grupos de falantes chineses (os recém-chegados e os que residem em Portugal por um período consideravelmente longo), mas difere do resultado obtido para a alveolar / $\mathrm{t}$ /, favorecedor em Yang e desfavorecedor neste.

A ausência de significância estatística para as variáveis extralinguísticas propostas (Nível de Proficiência em PB; Tempo de Contato com PB e Tempo de Residência no Brasil) motivou a observação dos resultados obtidos por participantes, o que foi possível pela computação da variável como aleatória na rodada performada pelo Rbrul. A Tabela 3 a seguir apresenta os resultados obtidos.

Tabela 3 - Participantes: respostas esperadas

\begin{tabular}{c|c|c|c|c}
\hline Fator & $\begin{array}{c}\text { Total de } \\
\text { Ocorrências }\end{array}$ & $\begin{array}{c}\text { Porcentagem de } \\
\text { Aplicação }\end{array}$ & $\begin{array}{c}\text { Log- } \\
\text { odds }\end{array}$ & $\begin{array}{c}\text { Peso } \\
\text { Relativo }\end{array}$ \\
\hline $\mathrm{V}$ & 144 & $87,5 \%$ & 0,571 & 0,641 \\
\hline $\mathrm{C}$ & 144 & $84,7 \%$ & 0,373 & 0,594 \\
\hline $\mathrm{W}$ & 143 & $83,2 \%$ & 0,27 & 0,569 \\
\hline $\mathrm{F}$ & 144 & $68,1 \%$ & $-0,548$ & 0,368 \\
\hline $\mathrm{H}$ & 144 & $64,6 \%$ & $-0,709$ & 0,332 \\
\hline \hline TOTAL & 719 & $77,6 \%$ & \multicolumn{3}{l}{} \\
\hline \multicolumn{2}{|c|}{ Fonte: Tso (2016), por meio do programa computacional Rbrul (JOHNSON, 2016) }
\end{tabular}

Dos 05 participantes considerados na amostra, 03 apresentaram desempenho favorável, com pesos relativos de 0,641 (participante V), de 0,594 (participante C) e 0,569 (participante W), e dois, desfavorável, com pesos de 0,368 (participante F) e de 0,332 (participantes H). A observação das características extralinguísticas expressas no Quadro 1 anterior revela que o tempo de imersão linguística é importante para o processo de aquisição da LNN, mas não ocupa um papel decisivo, pois o participante $\mathrm{W}$, que apresenta maior tempo de imersão linguística, de aproximadamente 8 anos, não mostrou o melhor desempenho. $O$ participante $F$, que viveu no Brasil por aproximadamente três anos, apresentou peso relativo abaixo de 0,5. O participante $\mathrm{C}$, apesar de ter residido no Brasil por somente dois meses, apresentou desempenho superior ao do participante W. É importante ressaltar que C teve uma experiência de estudo em Portugal por seis meses; dessa forma, é possível pensar que ele esteja mais familiarizado com a língua portuguesa em comparação ao participante $H$.

Quanto ao nível de proficiência em língua portuguesa, dentre os participantes que obtiveram melhores resultados nos testes estão $\mathrm{W}$ e $\mathrm{V}$, os dois participantes com nível avançado. Ainda que o participante C (intermediário para avançado) apresente um desempenho melhor do 
que o participante W (avançado), a diferença é pequena, de 0,025, a menor observada entre os pesos relativos da Tabela 3 .

A análise por participante corroborou a irrelevância estatística da variável Tempo de contato com o PB revelada pelo Rbrul. O participante com melhor desempenho, a saber V, é o que apresenta o menor tempo, de 30 meses; o participante com mais tempo, de 96 meses, é aquele que apresenta o segundo melhor desempenho.

Quando considerado apenas o desempenho dos participantes nos dois testes com logatomas, os resultados apontaram novamente para a pouca relevância do tempo de imersão linguística para a amostra. De acordo com os resultados apresentados na Tabela 4 a seguir, os participantes $\mathrm{V}$ e F, que apresentam tempo de imersão linguística muito próximos, de 30 e de 36 meses, respectivamente, revelaram resultados bem divergentes, sendo que o peso relativo do participante $\mathrm{V}$ está acima do ponto neutro $(0,656)$ e o do participante $\mathrm{F}$, abaixo do ponto neutro $(0,316)$. Por outro lado, o nível de proficiência correlacionou-se positivamente ao desempenho na tarefa com logatomas: os falantes que possuem o nível mais avançado (os participantes $\mathrm{V}$ e $\mathrm{W}$ ) apresentaram desempenhos melhores em comparação aos outros participantes.

Tabela 4 - Identificação de Plosivas: respostas esperadas por Participante - teste Logatomas

\begin{tabular}{c|c|c|c|c}
\hline Fator & $\begin{array}{c}\text { Total de } \\
\text { Ocorrências }\end{array}$ & $\begin{array}{c}\text { Porcentagem de } \\
\text { Aplicação }\end{array}$ & Log-odds & $\begin{array}{c}\text { Peso } \\
\text { Relativo }\end{array}$ \\
\hline $\mathrm{V}$ & 72 & $83,3 \%$ & 0,638 & 0,656 \\
\hline $\mathrm{W}$ & 71 & $74,6 \%$ & 0,243 & 0,562 \\
\hline $\mathrm{C}$ & 72 & $73,6 \%$ & 0,196 & 0,551 \\
\hline $\mathrm{H}$ & 72 & $59,7 \%$ & $-0,339$ & 0,418 \\
\hline $\mathrm{F}$ & 72 & $47,2 \%$ & $-0,779$ & 0,316 \\
\hline \hline TOTAL & 359 & $67,7 \%$ & & \\
\hline Fonte: Tso (2016), por meio do programa computacional Rbrul (JOHNSON, 2016)
\end{tabular}

Embora a variável Vogal Seguinte não tenha sido apontada como estatisticamente relevante, foi construído o Gráfico 1 a seguir, que apresenta o cruzamento entre essa variável e Ponto de Articulação, a fim de que se possa verificar o papel da coarticulação entre consoante e $\operatorname{vogal}(/ \mathrm{o} / \mathrm{e} / \mathrm{a} /$, especificamente) na percepção das plosivas do $\mathrm{PB}$ por falantes do mandarim.

\section{Gráfico 1 - Ponto de Articulação da Consoante e Qualidade da Vogal Seguinte}

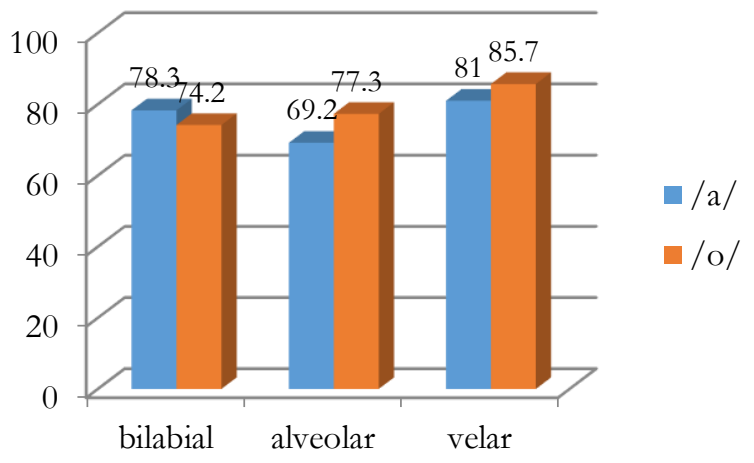

Fonte: Tso (2016), por meio do programa computacional Rbrul (JOHNSON, 2016)

No gráfico anterior, observa-se que as diferenças, em porcentagem, entre os três pares contrastivos é pequena, ou seja, para bilabiais, alveolares e velares é pequena a diferença de desempenho na percepção dos segmentos oclusivos seguidos por $/ \mathrm{a} /$ ou por $/ \mathrm{o} /$, sendo comparativamente maior para as alveolares (de 8,1 pontos percentuais) e praticamente idêntica para bilabiais (4,1 pontos percentuais) e velares (4 pontos percentuais). 
Verifica-se, desse modo, que os participantes apresentaram mais facilidade na identificação das plosivas adjacentes à vogal /o/ em comparação à vogal /a/, resultado que contraria a hipótese de que, por ser produzida com a cavidade oral mais aberta, sua produção facilitaria a identificação dos segmentos oclusivos a ela adjacentes.

Com relação a esse resultado, soma-se o levantamento de palavras desconhecidas pelos participantes expresso na Tabela 5 a seguir, obtido mediante a aplicação de questionário durante a coleta de dados. Dentre as 25 palavras desconhecidas, 16 (64\%) envolveram estímulos que apresentam a vogal /a/ após a plosiva em análise.

Tabela 5 - Palavras Desconhecidas destacadas pelos Participantes

\begin{tabular}{|c|c|c|c|c|c|c|c|c|c|}
\hline \multicolumn{2}{|c|}{$/ \mathrm{p} /$} & \multicolumn{2}{c|}{$/ \mathrm{b} /$} & \multicolumn{2}{c|}{$/ \mathrm{t} / \mathrm{c} / \mathrm{k} /$} & \multicolumn{2}{c|}{$/ \mathrm{g} /$} \\
\hline /a/ & $/ \mathrm{o} /$ & $/ \mathrm{a} /$ & $/ \mathrm{o} /$ & $/ \mathrm{a} /$ & $/ \mathrm{o} /$ & $/ \mathrm{a} /$ & $/ \mathrm{o} /$ & $/ \mathrm{a} /$ & $/ \mathrm{o} /$ \\
\hline $\begin{array}{c}\text { papa } \\
\text { pata }\end{array}$ & poço & $\begin{array}{c}\text { baba } \\
\text { batalha } \\
\text { dadivar } \\
\text { datar } \\
\text { datador }\end{array}$ & $\begin{array}{c}\text { boba } \\
\text { bocejar } \\
\text { bochecha }\end{array}$ & $\begin{array}{c}\text { tabaco } \\
\text { tacar } \\
\text { tapete }\end{array}$ & $\begin{array}{c}\text { topo } \\
\text { tolo } \\
\text { torrar }\end{array}$ & cachaça & - & $\begin{array}{c}\text { alga } \\
\text { gaguejo } \\
\text { galo } \\
\text { gata } \\
\text { ruga }\end{array}$ & $\begin{array}{c}\text { goleiro } \\
\text { gozo }\end{array}$ \\
& & & & & & & \\
\hline
\end{tabular}

Dado o levantamento de dados expresso na Tabela 5 anterior, em que um número maior de palavras com vogal /o/ seguinte à plosiva é reconhecido pelos participantes em comparação a palavras com vogal /a/, é razoável pensar que o significado exerce papel importante no processo perceptual; ou, de outro modo, a sequência de segmentos fonológicos relacionada a um significado parece exercer influência relevante no plano de reconhecimento de um segmento específico dessa sequência. Tal resultado é corroborado pelo fato de que a variável Vogal Seguinte não foi selecionada como estatisticamente relevante para a percepção dos segmentos oclusivos quando consideradas para computação estatística apenas as respostas provenientes dos testes com logatomas.

\section{CONSIDERAÇÕES FINAIS}

Este trabalho investigou o processo de percepção das consoantes plosivas /p, b, t, d, k, g/ do PB como LNN por cinco falantes nativos de mandarim. A principal distância entre o sistema de plosivas das duas línguas diz respeito ao contraste de sonoridade, ausente em mandarim.

De acordo com os resultados obtidos nos testes de percepção, foi possível verificar que a imersão linguística não apresentou papel decisivo. Já o nível de proficiência em LNN mostrou-se relevante, visto que falantes mais proficientes apresentaram melhor desempenho nos testes. Tal resultado corrobora o proposto em Flege (1995), para quem o sistema fonético e fonológico permanece adaptativo durante a vida dos falantes, pois cada vez em que é adicionado a esses sistemas um novo segmento vocálico ou consonantal, ocorrem recomposições. Embora os segmentos da L1 e da LNN encontrem-se em um mesmo plano fonológico na mente do falante na fase inicial de contato com a LE, à medida que o falante interage com a LNN e que se familiariza mais com a língua, a capacidade de diferenciar os segmentos das duas línguas aumenta.

Com relação aos fatores linguísticos, pode-se afirmar que a coarticulação entre as plosivas /p, b, t, d, k, g/ e as vogais /a/ e /o/ não interferiu na percepção dos participantes. Embora os resultados dos testes de palavras reais tenham apresentado a vogal /o/ como condição favorável à identificação das plosivas, o papel desempenhado pelo conhecimento do significado das palavras pelos participantes mostrou-se mais relevante.

Quanto à identificação das plosivas, os resultados indicam que os participantes tiveram mais facilidade na distinção do par $/ \mathrm{k} /-/ \mathrm{g} /$, seguido por $/ \mathrm{p} /-/ \mathrm{b} / \mathrm{e} / \mathrm{t} /-/ \mathrm{d} /$. De modo geral, os participantes deste estudo apresentaram um bom domínio na distinção das plosivas, com 
resultados individuais pouco discrepantes. Porém, não é possível concluir que os falantes não tenham dificuldades na distinção dos segmentos tratados, pois no segundo questionário aplicado na última etapa da coleta de dados, todos admitiram uma grande dificuldade durante a realização dos testes, principalmente dos testes com logatomas. Vale registrar que, embora a opção Não Sei estivesse presente para todos os testes, apenas um participante optou por clicá-la por somente uma vez; ou seja, não foi possível evitar escolhas aleatórias nos testes, mas, de qualquer forma, houve claramente empenho por parte de todos para a escolha da opção que mais se assemelhou ao segmento apresentado.

O bom desempenho nos testes parece estar também relacionado ao grau de imersão na LNN. De acordo com o primeiro questionário respondido, os participantes declararam que não costumam viver em comunidade fechada e formada somente por chineses, mas que procuram sair com os colegas brasileiros, assistir aos filmes dublados, ouvir rádio e ler revistas em português.

É preciso salientar que este estudo considerou um número pequeno de participantes, com níveis de proficiência em PB aproximados, de intermediário a avançado. Replicar a proposta a um grupo experimental mais amplo, com diferentes níveis de proficiências, poderá complementar a análise apresentada.

\section{REFERÊNCIAS BIBLIOGRÁFICAS}

BARBOSA, P. A.; MADUREIRA, S. Manual de fonética acústica experimental. São Paulo: Cortez, 2015.

BEST, C. A direct realist view of cross-language speech perception. In: STRANGE, Winifred. Speech perception and linguistic experience. Issues in cross-language research. Timonuim, MD: York Press, 1995. p. $171-204$.

BEST, C.; TYLER, M. Nonnative and second-language speech perception: commonalities and complementarities. In: MUNRO, Murray; BOHN, OCKE-Schwen. Language experience in second language speech learning: in honor of James Emil Flege. Amesterdam/ Philadelphia: John Benjamin's Publishing Company, 2007. p. 13 - 34.

BOERSMA, P.; WEENINK, D. Praat: doing phonetics by computer [Programa de computador]. Versão 6.0.21, 2016.

CLEMENTS, N. The role of the sonority cycle in core syllabification. Working Papers of the Cornell Phonetics Laboratory, n. 2, p. 1-68, abr. 1990.

FLEGE, J. E. The phonetic study of bilingualism. In: G. Bloothooft, V. Hazan, D. Huber, J. Llisterri (Eds.). European Studies in Phonetics and Speech Communication. Utrecht: OTS, 1995. p. 98-103.

FLEGE, J. E. Second language speech learning theory, findings, and problems. In: STRANGE, Winifred. Speech perception and linguistic experience: issues in cross-language research. Timonuim, MD: York Press, 1995.

JESPERSEN, O. Lehrbuch der Phonetik. Leipzig, Berlin: Teubner, 1904.

HOOPER, J. B. An introduction to natural generative phonology. New York: Academic Press, 1976. 
HUANG, B.R; LI, W. Xiandai Hanyu. Beijing: Peking University Press, 2012.

PROGRAMA DE PORTUGUÊS PARA ESTRANGEIRO - UFRGS. Disponível em <http://www.ufrgs.br/ppe/o-programa>. Acesso em: 24 out. 2016.

SILVA, S. M. Aprendizagem fonológica e alofônica em L2: percepção e produção das vogais médias do português por falantes nativos do espanhol. 2014. Tese (Doutorado em Letras) Pontifícia Universidade Católica do Rio Grande do Sul, Porto Alegre.

TATHAM, M.; MORTON, K. Speech production and perception. U.S.: Palgrave Macmillan, 2006.

TSO, F. W.C. A percepção das consoantes oclusivas do Português Brasileiro como LE por falantes nativos do mandarim, 2016 (Trabalho de Conclusão do Curso) - Pontifícia Universidade Católica do Rio Grande do Sul, Porto Alegre, 2016.

YANG, S. Percepção das consoantes oclusivas de português L2 Sob a influência de mandarim L1. 2014. Dissertação (Doutorado em Letras) - Universidade do Minho, Braga.

YUAN, J.H. Hanyu Fangyan Gaiyao. 2. ed. Beijing: YuWen Audio and Video Publishing Press, 2001.

Submetido em 14/11/2018

Aceito em 08/04/2019

Publicado em 26/04/2019 